\title{
BIOCONVERSIÓN DE DESPERDICIOS VEGETALES A BIOGÁS A PARTIR DE MICROORGANISMOS RUMINALES
}

\author{
Rafael Germán CAMPOS-MONTIEL ${ }^{1}$, Óscar Enrique DEL RAZO-RODRÍGUEZ ${ }^{1}$, \\ Isaac ALMARAZ-BUENDÍA ${ }^{1 *}$, Efrén RAMÍREZ-BRIBIESCA ${ }^{2}$, Ramón SORIANO-ROBLES ${ }^{3}$, \\ Jesús Armando SALINAS-MARTÍNEZ ${ }^{1}$, Ladislao ARIAS-MARGARITO ${ }^{3}$ y \\ Sergio Segundo GONZÁLEZ-MUÑOZ ${ }^{2}$
}

${ }^{1}$ Instituto de Ciencias Agropecuarias, Universidad Autónoma del Estado de Hidalgo, Rancho Universitario, Av. Universidad km. 1, Ex-Hda. de Aquetzalpa AP 32, 43600 Tulancingo, Hidalgo, México

${ }^{2}$ Colegio de Postgraduados, Campus Montecillo, Carretera México-Texcoco km. 36.5, Montecillo, 56230 Texcoco, Estado de México, México

${ }^{3}$ Biología de la Reproducción, Universidad Autónoma Metropolitana-Iztapalapa, San Rafael Atlixco 186, Col. Vicentina, 09340 Iztapalapa, México

*Autor de correspondencia; isac@xanum.uam.mx

(Recibido enero 2017; aceptado junio 2017)

Palabras clave: metano, desechos orgánicos, producción de gas, bioenergía, residuos vegetales

\section{RESUMEN}

La creciente demanda de alimentos en áreas urbanas genera cada vez mayor cantidad de desperdicios orgánicos, incluyendo residuos vegetales. El manejo de estos residuos vegetales causa emisiones de gases efecto invernadero (GEI) por su recolección, transporte, y depósito en vertederos. Para identificar su potencial de GEI, en un mercado de alimentos se recolectaron residuos de hojas de lechuga (LS), de col (BOV), de coliflor (BOB), de maíz (HZM), de espinaca (SO) y de nopal (OF), se fermentaron in vitro usando líquido ruminal como inóculo y se compararon con heno de avena (AS), heno de alfalfa $(\mathrm{AH})$ y ensilado de maíz (EZM), forrajes utilizados con frecuencia en la alimentación de rumiantes. Las variables fueron degradación in vitro de la materia seca (DIVMS), pH, cinética de producción de gas y concentración de metano $\left(\mathrm{CH}_{4}\right)$. La fermentación se realizó durante $96 \mathrm{~h}$ a $39^{\circ} \mathrm{C}$ y las muestras de gas para medir la concentración de $\mathrm{CH}_{4}$ se tomaron a 9, 12, 24, 34 y 48 h de incubación. La DIVMS de LS, BOV, BOB, HZM y OF fue $12.8 \%$ mayor a AS, AH y EZM. Entre las 9 y $12 \mathrm{~h}$ de incubación la emisión de $\mathrm{CH}_{4}$ a partir de $\mathrm{BOV}$, BOB and $\mathrm{HZM}$ fue $44.8 \%$ superior a los tres forrajes. La principal conclusión es que estos residuos vegetales son degradados adecuadamente por los microorganismos ruminales y, por lo tanto, se pueden usar para la producción de biogás en áreas urbanas para disminuir su depósito en vertederos.

Key words: methane, organic wastes, gas production, bioenergy, vegetable residues

\begin{abstract}
The growing demand for food in urban areas generates an increasing amount of organic wastes, including vegetable residues. The management of these wastes can lead to greenhouse gases emissions (GHG) through its process, including collection, transportation,
\end{abstract}


and accumulation in landfills. To identify the potential GHG from vegetable residues, in vitro fermentations of collected residues of lettuce (LS), cabbage (BOV), cauliflower (BOB), corn cob (HZM), and spinach (SO) leaves, as well as leftovers of cladodes from cactus pear (OF) from a food market, were compared with oat hay (AS), lucerne hay $(\mathrm{AH})$ and corn silage (EZM), major components in ruminant feed using rumen fluid as the inoculum. The variables were: in vitro dry matter degradation (IVDMD), $\mathrm{pH}$, kinetics of gas production and methane $\left(\mathrm{CH}_{4}\right)$ concentration. Fermentation was carried out at $39{ }^{\circ} \mathrm{C}$ for $96 \mathrm{~h}$, and the gas samples to measure $\mathrm{CH}_{4}$ concentration were taken at $9,12,24,34$ and $48 \mathrm{~h}$ of incubation. The IVDMD of LS, BOV, BOB, HZM and OF was $12.8 \%$ higher than those of the forages (AS, AH and EZM). Between 9 and $12 \mathrm{~h}$ of incubation, $\mathrm{CH}_{4}$ emission from $\mathrm{BOV}, \mathrm{BOB}$ and $\mathrm{HZM}$ was $44.8 \%$ higher than that from the three forages. Our results suggest that vegetable residues can be adequately degraded by the rumen microorganisms and, therefore, they can be used for biogas production in urban areas to decrease their accumulation in landfills.

\section{INTRODUCCIÓN}

Los desperdicios de alimentos de origen vegetal se generan durante la distribución y comercialización de alimentos para consumo humano (FAO 2015) y se incrementan con los niveles de la población y con los cambios en los estándares de vida (Zebek et al. 2015). Estos desperdicios se acumulan en grandes cantidades en mercados y tiendas de autoservicio en todo el mundo, lo que incrementa costos por operación, transporte y deposición (Scano et al. 2014). La Central de Abastos de la Ciudad de México es considerada el mercado más grande de alimentos de América Latina porque produce $585 \mathrm{t} / \mathrm{d}$ de residuos sólidos, de los cuales aproximadamente $80 \%$ son orgánicos; esto genera gastos en combustible, emisiones de bióxido de carbono (SEDEMA 2015) y de $\mathrm{CH}_{4}$, tanto por su descomposición como por su traslado. Parte de estos desperdicios vegetales se usan como suplemento en los sistemas pecuarios que existen en los espacios urbanos y periurbanos (Losada et al. 1998, 2000); el resto se deposita en vertederos a pesar de ser productos perecederos (FAO 2015). La digestión anaerobia de estos desperdicios en los vertederos produce una gran cantidad de biogás que contiene de 40 a $70 \%$ de $\mathrm{CH}_{4}$ y que se emite a la atmósfera, lo cual, contribuye al calentamiento global (Thi et al. 2014).

La digestión anaerobia es una tecnología disponible para producir energía renovable, reducir las emisiones de gases de efecto invernadero (GEI), generar energía a partir de recursos renovables e incrementar la eficiencia energética a partir de sustratos de bajo costo, como son desperdicios de frutas y vegetales (di Maria et al. 2015, Li et al. 2016, Yan et al. 2017). El manejo adecuado de desperdicios y residuos vegetales puede generar sistemas producti- vos integradores (Zebeck et al. 2015) y mejorar las condiciones ambientales al capturar este biogás y usarlo como sustituto de combustibles fósiles (Cioabla et al. 2013, FAO 2015). Con base en lo anterior, el objetivo fue comparar el volumen de $\mathrm{CH}_{4}$ generado por fermentación in vitro de algunos desperdicios vegetales con el generado por heno de avena, heno de alfalfa y ensilado de maíz, usando líquido ruminal como inóculo.

\section{MATERIALES Y MÉTODOS}

El presente estudio se realizó en los laboratorios de Nutrición Animal y de Análisis Especiales del Instituto de Ciencias Agropecuarias en Universidad Autónoma del Estado de Hidalgo (UAEH). Los desperdicios vegetales se obtuvieron en la Central de Abastos de Ecatepec, Estado de México; el heno de avena (Avena sativa, AS), heno de alfalfa (Medicago sativa, AH) y ensilado de maíz (EZM), en el rancho universitario de la UAEH. Los desperdicios vegetales se seleccionaron por volumen y se tomaron muestras representativas de lechuga Lactuca sativa (LS), col Brassica oleraceae var. viridis (BOV), coliflor Brassica oleraceae var. botrytis (BOB), hojas de maíz Zea mays, (ZM), hojas y parte del tallo de espinacas Spinacia oleraceae (SO) y nopal Opuntia ficus (OF) de distintos locales comerciales. Las muestras se secaron a $55{ }^{\circ} \mathrm{C}$ por $48 \mathrm{~h}$ en estufa de aire forzado (Riossa, México) y se molieron (Thomas-Willey, Philadelphia, Pa, EUA) usando una criba de $1 \mathrm{~mm}$ de diámetro. De las muestras se analizó materia seca (MS, método 930.15), materia orgánica (MO) y cenizas (método 942.05) de acuerdo con las especificaciones de la Association of Official Analytical Chemists (AOAC, 1990). 


\section{Fermentación in vitro}

Se usó el método descrito por Theodorou et al. (1994). En frascos de vidrio $(120 \mathrm{ml})$ se depositaron $0.5 \mathrm{~g}$ de MS de una muestra (cinco repeticiones independientes). Dos frascos sin sustrato se usaron como blancos. El líquido ruminal se extrajo de tres ovinos antes de alimentarlos con avena henificada y agua a libre acceso, se filtró a través de cuatro capas de gasa y se almacenó a $39^{\circ} \mathrm{C}$ en condiciones anaerobias hasta su uso. A cada frasco y con flujo continuo de $\mathrm{CO}_{2}$ se le agregaron $40 \mathrm{ml}$ de medio líquido compuesto por $8.99 \mathrm{ml}$ de solución amortiguadora ( $\left.4 \mathrm{~g} \mathrm{NH}_{4} \mathrm{HCO}_{3} / \mathrm{L}_{\text {y }} 35 \mathrm{~g} \mathrm{NaHCO}_{3} / \mathrm{L}\right), 8.99 \mathrm{ml}$ de solución macro mineral $\left(5.7 \mathrm{~g} \mathrm{Na}_{2} \mathrm{HPO}_{4} / \mathrm{L}, 6.2 \mathrm{~g}\right.$ $\mathrm{KH}_{2} \mathrm{PO}_{4} / \mathrm{L}$ y $\left.0.6 \mathrm{~g} \mathrm{MgSO}_{4} \cdot 7 \mathrm{H}_{2} \mathrm{O} / \mathrm{L}\right), 4.6 \mu \mathrm{l}$ de solución micro mineral $\left(132 \mathrm{~g} \mathrm{CaCl}_{2} \cdot 2 \mathrm{H}_{2} \mathrm{O} / \mathrm{L}, 100 \mathrm{~g}\right.$ $\mathrm{MnCl} \cdot 4 \mathrm{H}_{2} \mathrm{O} / \mathrm{L}, 10 \mathrm{~g} \mathrm{CoCl} \cdot 6 \mathrm{H}_{2} \mathrm{O} / \mathrm{L}$ y $80 \mathrm{~g} \mathrm{FeCl} / \mathrm{L}$ ), $0.05 \mathrm{ml}$ de resazurina ( $1 \mathrm{~g}$ Resazurin/L), $17.97 \mathrm{ml}$ de agua destilada y $4 \mathrm{ml}$ de líquido ruminal. Los frascos se sellaron herméticamente (engargoladora manual, Wheaton, EUA) mediante un tapón siliconizado y una cápsula vial con centro desprendible y se incubaron a $39^{\circ} \mathrm{C}$ por $72 \mathrm{~h}$ en baño maría (Thermo Scientific, Mod. 2864, EUA). La producción de gas se midió por desplazamiento de volumen de agua insertando una aguja $(18 \mathrm{G} \times 38 \mathrm{~mm}$ conectada mediante una manguera de látex a una bureta de $50 \mathrm{ml}$ llena de $\mathrm{H}_{2} \mathrm{O}$ destilada) en cada frasco a las $0,1,2,3,5,7,9,12$, $16,20,24,34,48,58$ y 72 h de incubación. El gas se liberó después de cada lectura y se ajustó a ml/g MS. El pH se midió (HANNA, HI2211, Rumania) al terminar el tiempo de incubación y el contenido de cada frasco se transfirió a tubos de polisulfona de $50 \mathrm{ml}$. Los tubos se centrifugaron (HERMLE, Z326K, Alemania) a $19461 \times \mathrm{g}$ por $15 \mathrm{~min}$, la fracción líquida se desechó y los tubos se secaron en un horno (Lumistell, HTP-41, México) a $60{ }^{\circ} \mathrm{C}$ por $48 \mathrm{~h}$. La degradación in vitro de la materia seca (DIVMS) se obtuvo por diferencia entre la materia seca al inicio y al final de la incubación (Theodorou et al. 1994).

Las variables de producción de gas se obtuvieron utilizando el modelo logístico (Schofield et al. 1994) y el procedimiento NLIN (SAS 2003):

$\mathrm{F}(t)=v / 1+\mathrm{e}^{2-4 r(\lambda-t)}$

donde $v$ es el volumen máximo de gas, $r$ la tasa de degradación del sustrato y $\lambda$ el tiempo de retardo.

\section{Concentración de $\mathrm{CH}_{4}$}

Tres mililitros de gas de cada frasco se extrajeron con jeringas desechables de $5 \mathrm{ml}$ y aguja de $21 \mathrm{G} \times$ $32 \mathrm{~mm}$ antes de cada lectura a las 9, 12, 24, 34 y $48 \mathrm{~h}$ de incubación. El gas se inyectó inmediatamente en frascos serológicos $(10 \mathrm{ml}$, sellados herméticamente con tapón de silicón y arillo de aluminio) desplazando la solución salina saturada $(6 \mathrm{~N}, \mathrm{pH} 2$ y rojo de metilo como indicador) de su interior. Los frascos se almacenaron durante una semana a temperatura ambiente con la tapa hacia abajo y se inyectaron (jeringa de vidrio, Hamilton CO., Reno, Nevada, EUA) $0.2 \mathrm{ml}$ del gas en un cromatógrafo de gases (Perkin Elmer, Autosystem XL, EUA) equipado con metanizador, detector de ionización de flama (FID) y columna capilar (Elite Plot Q $30 \mathrm{~m} \times 0.53 \mathrm{~mm}$, series GC). La temperatura del detector fue de $200{ }^{\circ} \mathrm{C}$, la del inyector de $200^{\circ} \mathrm{C}$ y la del horno de $50^{\circ} \mathrm{C}$, con un tiempo de corrida de $5 \mathrm{~min}$. La concentración de $\mathrm{CH}_{4}$ se calculó usando estándares 30:70, 50:50 y 100:00 de $\mathrm{CH}_{4}: \mathrm{CO}_{2}$; por su parte, el volumen de $\mathrm{CH}_{4} / \mathrm{g}$ $\mathrm{MS} / \mathrm{h}$ se calculó dividiendo la cantidad de gas entre las horas transcurridas entre cada lectura, salvo de 12 a 24 h donde sólo se consideró el gas acumulado de las 16 a las $24 \mathrm{~h}$.

\section{Análisis estadístico}

Las variables se analizaron con un diseño completamente aleatorio. El modelo estadístico fue el siguiente:

$y_{i j}=\mu+\beta_{i}+\varepsilon_{i j} \quad \imath: 1,2,3$

donde $y_{\mathrm{ij}}$ es la variable respuesta, $\mu$ la media general, $\beta i$ el efecto del sustrato y $\varepsilon_{\mathrm{ij}}$ el error aleatorio. Los datos se analizaron con el procedimiento GLM (SAS 2003) y la comparación de medias con la prueba de Tukey ( $\leq 0.05)$.

\section{RESULTADOS Y DISCUSIÓN}

\section{Fermentación in vitro}

Los desperdicios de vegetales y frutas contienen alrededor de $75 \%$ de materia orgánica que puede degradarse por digestión anaerobia (Li et al. 2016, Sánchez-Reyes et al. 2016). La DIVMS de HZM, $\mathrm{BOV}, \mathrm{BOB}, \mathrm{LS}$ y OF fue $12.89 \%$ mayor $(\mathrm{p} \leq 0.05)$ comparada con AS, AH, y EZM (Cuadro I). Esto sugiere que los microrganismos ruminales degradan adecuadamente desperdicios vegetales (a excepción de SO) después de $72 \mathrm{~h}$ de incubación en las condiciones descritas. Sin embargo, la digestión anaerobia es afectada por la temperatura, $\mathrm{pH}$, relación sólidoslíquidos, tipo de sustrato (Li et al. 2016, Sen et al. 2016), y tipo y densidad del inóculo (Islas-Espinosa et al. 2017). En este contexto, en el presente estudio 
se proporcionaron las condiciones óptimas para los microorganismos ruminales de acuerdo con la metodología descrita por Theodorou et al. (1994).

La digestibilidad del sustrato es factor clave para generar biogás. La DIVMS de la mayoría de los desperdicios vegetales fue superior a $60 \%$. La biodegradabilidad de SO, LS y BOB inoculados con lodo anaerobio fue de 42, 49 y $55 \%$ (Yan et al. 2017) y la DIVMS de OF inoculado con líquido ruminal fue superior a $70 \%$ (del Razo et al. 2015). Sauer et al. (2012) argumentan que el ecosistema ruminal puede ser una alternativa para la bioconversión de material lignocelulósico en combustibles y químicos, lo cual concuerda con la DIVMS inoculada con líquido ruminal. El pH en el medio líquido al final de la incubación fue menor $(p<0.05)$ para HZM, lo que explica su mayor DIVMS y V (Cuadro I) ya que, a mayor digestibilidad, mayor liberación de iones hidrógeno. Sin embargo, la fermentación de LS, BOV, BOB y OF no provocó los mismos efectos, lo que sugiere un efecto acidificante menor que se relaciona con bajo contenido de azúcares solubles (Scano et al. 2014, Li et al. 2016). No obstante, a pesar de los cambios en el pH al final de la incubación, éste fue de 6.5 a 7, el cual está dentro del rango ideal (6.5 a 7.5) para los microorganismos metanogénicos (Sen et al. 2016) y es similar a lo reportado por Sánchez-Reyes et al. (2016) con valores de 6.94 a 7.95 en digestión anaerobia de frutas y vegetales.

A pesar que la DIVMS de LS fue similar $(\mathrm{p}>0.05)$ a BOV, BOB, HZM y OF, el V fue menor ( $\mathrm{p} \leq 0.05$ ) para los mismos tratamientos; pero similar a SO y AH. De acuerdo con Schofield et al. (1994) y Theodorou et al. (1994), la producción de gas está altamente correlacionada con la DIVMS, por lo que estos desperdicios vegetales se degradaron mejor $(60 \%)$ que los forrajes $(55 \%)$. Estos resultados son similares a lo reportado por Islas-Espinosa et al. (2017), quienes cuantificaron digestibilidades de 55 a $65 \%$ de MS y de 200 a $300 \mathrm{ml} / \mathrm{g}$ MS de biogás con desperdicios vegetales usando excretas como inóculo. Lo anterior indica que tanto excretas como líquido ruminal sirven como inóculo en la digestión anaerobia de desperdicios vegetales sin que se afecte la degradación de la MS.

El valor de $r$ (tasa de degradación del sustrato) fue mayor $(\mathrm{p} \leq 0.05)$ para $\mathrm{OF}$ seguido por BOV y $\mathrm{BOB}$, indicando que estos sustratos son degradados rápidamente por microrganismos ruminales. Yan et al. (2017) también reportaron mayor tasa de degradación y tiempo de retardo en BOB fermentando hojas de distintos vegetales. El tiempo de retardo $(\lambda)$ fue mayor para EZM seguido por OF y BOB, por lo que los microorganismos ruminales tardan más en adherirse a estos sustratos.

\section{Concentración de $\mathrm{CH}_{4}$}

Las hojas de HZM, BOV, BOB y AH produjeron mayor cantidad $(\mathrm{p} \leq 0.05)$ de $\mathrm{CH}_{4}$ de las 7 a las $9 \mathrm{~h}$ de incubación (Cuadro II) seguidas por OF. La tendencia fue similar de las 9 a las $12 \mathrm{~h}$ de incubación para BOB, HZM y BOV (1.5 ml CH $4 /$ h; Fig. 1), los cuales generaron $44 \%$ más de $\mathrm{CH}_{4}$ en comparación con los tres forrajes. Hojas de variedades de coliflor y lechuga mostraron la mayor producción acumulada y máxima de $\mathrm{CH}_{4}$ a partir de su fermentación anaerobia (Yan et al. 2017), sugiriendo que HZM, BOB y $\mathrm{BOV}$ son referencia para posteriores estudios sobre producción de biogás.

CUADRO I. MATERIA ORGÁNICA, VARIABLES DE CINÉTICA DE PRODUCCIÓN DE GAS Y DESAPARICIÓN IN VITRO DE LA MATERIA SECA DE DESPERDICIOS VEGETALES Y DE TRES FORRAJES

\begin{tabular}{lcccccccccc}
\hline & LS & BOV & BOB & HZM & SO & OF & AS & AH & EZM & EE \\
\hline MS (\%) & 5.75 & 11.5 & 10.5 & 18.3 & 8.9 & 3.9 & 86.5 & 88.8 & 32.45 \\
MO (\%) & 76.3 & 83.7 & 85.9 & 96.7 & 77.2 & 81.74 & 91.1 & 89.1 & 11.46 & \\
Cenizas (\%) & 23.2 & 16.5 & 14.4 & 3.45 & 22.6 & 19.46 & 9.04 & 10.9 & 88.54 & \\
DIVMS (\%) & $62.06^{\mathrm{ab}}$ & $63.15^{\mathrm{a}}$ & $61.82^{\mathrm{ab}}$ & $63.71^{\mathrm{a}}$ & $56.00^{\mathrm{c}}$ & $63.62^{\mathrm{a}}$ & $54.22^{\mathrm{c}}$ & $57.91^{\mathrm{bc}}$ & $54.95^{\mathrm{c}}$ & 4.4 \\
pH & $6.98^{\mathrm{ab}}$ & $6.80^{\mathrm{cd}}$ & $6.85^{\mathrm{bcd}}$ & $6.44^{\mathrm{e}}$ & $7.04^{\mathrm{a}}$ & $6.92^{\mathrm{abc}}$ & $6.78^{\mathrm{cd}}$ & $6.93^{\mathrm{abc}}$ & $6.70^{\mathrm{d}}$ & 0.01 \\
V (ml) & $194.18^{\mathrm{fg}}$ & $273.20^{\mathrm{b}}$ & $261.42^{\mathrm{c}}$ & $327.17^{\mathrm{a}}$ & $184.40^{\mathrm{g}}$ & $222.10^{\mathrm{e}}$ & $245.22^{\mathrm{d}}$ & $205.82^{\mathrm{f}}$ & $251.65^{\mathrm{cd}}$ & 17.4 \\
r (-1) & $0.057^{\mathrm{d}}$ & $0.066^{\mathrm{b}}$ & $0.060^{\mathrm{c}}$ & $0.039^{\mathrm{ef}}$ & $0.040^{\mathrm{e}}$ & $0.069^{\mathrm{a}}$ & $0.034^{\mathrm{g}}$ & $0.060^{\mathrm{c}}$ & $0.036^{\mathrm{fg}}$ & $1.9 \mathrm{E}-6$ \\
$\lambda(\mathrm{h})$ & $0.367^{\mathrm{d}}$ & $-0.224^{\mathrm{e}}$ & $0.944^{\mathrm{c}}$ & $0.602^{\mathrm{cd}}$ & $0.384^{\mathrm{d}}$ & $1.44^{\mathrm{b}}$ & $0.321^{\mathrm{d}}$ & $0.370^{\mathrm{d}}$ & $3.14^{\mathrm{a}}$ & 0.02 \\
\hline
\end{tabular}

abcdefg Letras desiguales indican diferencias significativas en cada línea $(\mathrm{p}<0.05)$

LS: Lactuca sativa; BOV: Brassica oleraceae var. viridis; BOB: Brassica oleraceae var. botrytis; HZM: hojas de maíz; SO: Spinacia Oleraceae; OF: Opuntia ficus; AS: Avena sativa; AH: Medicago sativa; EZM: ensilado de maíz; MS: materia seca; MO: materia orgánica; DIVMS: digestibilidad in vitro de la materia seca; V: volumen máximo de gas; r: tasa de degradación del sustrato; $\lambda$ : tiempo de retardo; EE: error estándar 
CUADRO II. VOLUMEN DE $\mathrm{CH}_{4}$ GENERADO DURANTE LA FERMENTACIÓN IN VITRO DE DESPERDICIOS VEGETALES

\begin{tabular}{lllllllllll}
\hline $\mathrm{CH}_{4}(\mathrm{ml} / \mathrm{g} \mathrm{MS})$ & LS & BOV & BOB & HZM & SO & OF & AS & AH & EZM & EE \\
\hline Tiempo & & & & & & & & & & \\
7 a 9 & $2.41^{\text {ed }}$ & $3.88^{\mathrm{ab}}$ & $3.56^{\mathrm{ab}}$ & $4.06^{\mathrm{a}}$ & $2.10^{\mathrm{e}}$ & $3.31^{\mathrm{bc}}$ & $2.09^{\mathrm{e}}$ & $3.49^{\mathrm{ab}}$ & $2.75^{\mathrm{dc}}$ & 0.02 \\
$9 \mathrm{a} 12$ & $2.23^{\mathrm{de}}$ & $4.56^{\mathrm{a}}$ & $4.73^{\mathrm{a}}$ & $4.62^{\mathrm{a}}$ & $2.13^{\mathrm{e}}$ & $3.37^{\mathrm{bc}}$ & $2.79^{\mathrm{cd}}$ & $3.26^{\mathrm{bc}}$ & $3.53^{\mathrm{b}}$ & 0.02 \\
16 a 24 & $1.41^{\mathrm{c}}$ & $1.74^{\mathrm{c}}$ & $1.92^{\mathrm{c}}$ & $4.09^{\mathrm{a}}$ & $1.39^{\mathrm{c}}$ & $1.77^{\mathrm{c}}$ & $3.52^{\mathrm{b}}$ & $1.92^{\mathrm{c}}$ & $3.97^{\mathrm{ab}}$ & 0.02 \\
$24 \mathrm{a} 34$ & $1.45^{\mathrm{d}}$ & $1.48^{\mathrm{d}}$ & $1.86^{\mathrm{cd}}$ & $5.03^{\mathrm{a}}$ & $2.51^{\mathrm{c}}$ & $1.79^{\mathrm{cd}}$ & $4.08^{\mathrm{b}}$ & $1.75^{\mathrm{cd}}$ & $4.68^{\mathrm{ab}}$ & 0.05 \\
$34 \mathrm{a} 48$ & $1.36^{\mathrm{b}}$ & $1.26^{\mathrm{b}}$ & $1.99^{\mathrm{b}}$ & $4.78^{\mathrm{a}}$ & $5.57^{\mathrm{a}}$ & $1.86^{\mathrm{b}}$ & $4.44^{\mathrm{a}}$ & $2.02^{\mathrm{b}}$ & $4.71^{\mathrm{a}}$ & 0.17 \\
\hline
\end{tabular}

abcde Letras desiguales indican diferencias significativas en cada línea $(\mathrm{p}<0.05)$

LS: Lactuca sativa; BOV: Brassica oleraceae var. viridis; BOB: Brassica oleraceae var. botrytis; HZM: hojas de maíz; SO: Spinacia Oleraceae; OF: Opuntia ficus; AS: Avena sativa; AH: Medicago sativa; EZM: ensilado de maíz; MS: materia seca; EE: error estándar 2.5

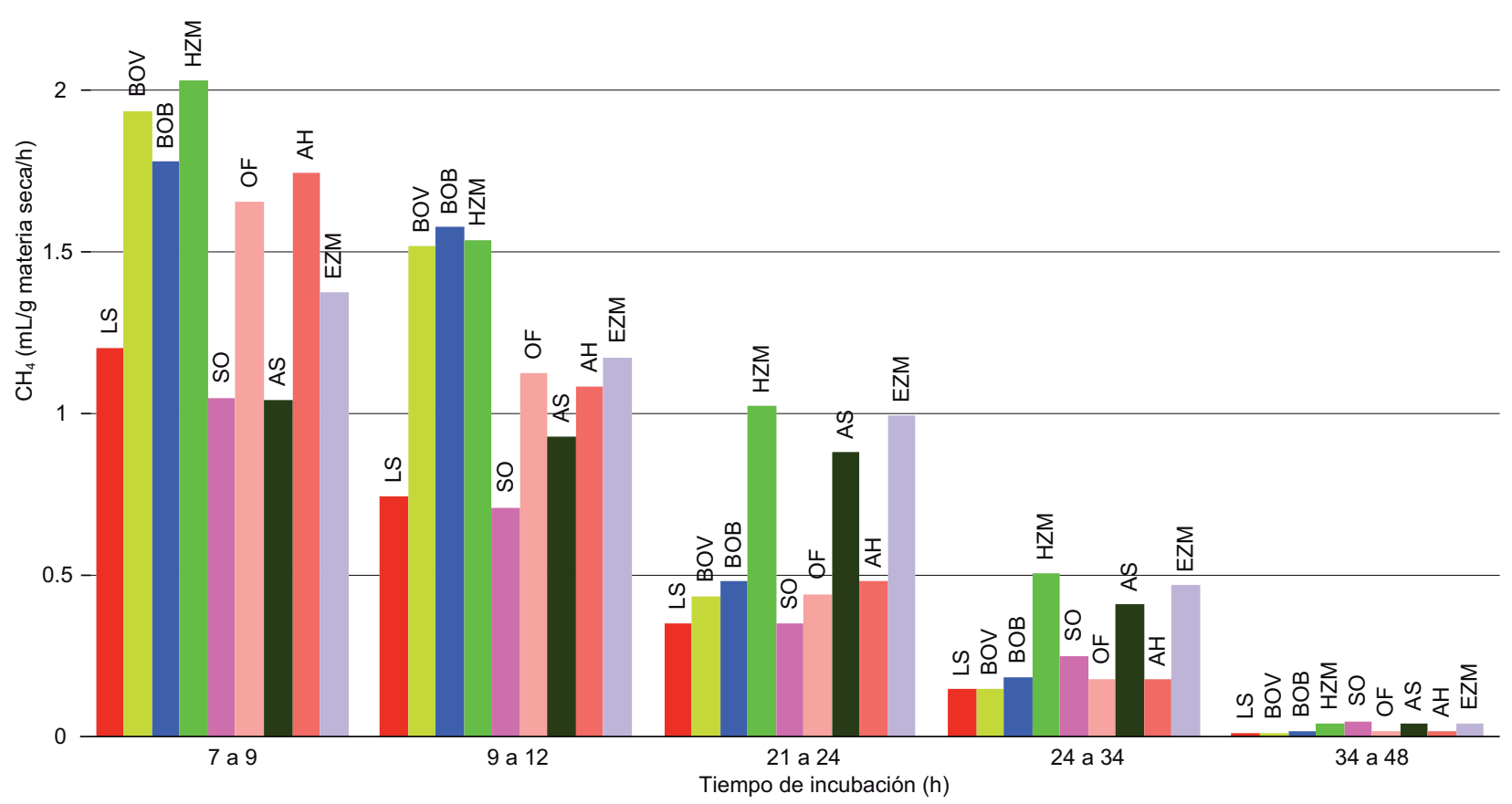

Fig. 1. Volumen de $\mathrm{CH}_{4}$ generado durante la fermentación in vitro de desperdicios vegetales con líquido ruminal como inóculo. LS: Lactuca sativa, BOV: Brassica oleraceae var. viridis, BOB: Brassica oleraceae var. botrytis, HZM: hojas de maíz, SO: Spinacia oleraceae, OF: Opuntia ficus, AS: Avena sativa, AH: Medicago sativa, EZM: ensilado de maíz

La acumulación de $\mathrm{CH}_{4}$ de las 16 a las $24 \mathrm{~h}$ de incubación fue mayor $(\mathrm{p} \leq 0.05)$ para HZM y EZM (1 ml CH $4 /$ h; Fig. 1); de las 24 a las 34 h, disminuyó a la mitad, seguido por AS (Fig. 1), que es un forraje de amplio uso en la alimentación de rumiantes. Finalmente, de las 34 a las $48 \mathrm{~h}$ de incubación, la acumulación de $\mathrm{CH}_{4}$ fue mayor para HZM, SO, AS y EZM $(0.05 \mathrm{ml} \mathrm{CH} / \mathrm{h})$, sin diferencia entre ellos.

Los resultados del presente estudio son superiores a los de Islas-Espinosa et al. (2017), quienes reportaron picos máximos de $0.9769 \mathrm{ml} \mathrm{CH}_{4} / \mathrm{g}$ MS entre las 8 y 10 h de digestión anaerobia, sugiriendo una mayor eficiencia de producción de $\mathrm{CH}_{4}$ por parte del líquido ruminal en las condiciones descritas. La emisión de $\mathrm{CH}_{4}$ entérico por los rumiantes contribuye de manera importante al calentamiento global (Bonilla y Lemus 2012), por lo que la generación de $\mathrm{CH}_{4}$ a partir de desperdicios vegetales y el generado a partir de forrajes comúnmente usados en la alimentación de rumiantes es punto de comparación. La producción 
de biogás a partir de desechos vegetales inoculados con líquido ruminal es una opción en las ciudades, porque los desechos o desperdicios vegetales que se generan con base en la creciente demanda de alimentos de la población tienen que conducir al desarrollo de una "cadena de uso" (Losada et al. 1998).

Los desperdicios de BOV, BOB, HZM y OF que se generan en grandes mercados de alimentos se pueden usar para producir $\mathrm{CH}_{4}$ por digestión anaerobia (di Maria et al. 2015, Yan et al. 2017). De acuerdo con Eriksson et al. (2015), la digestión anaerobia tiene gran potencial para reducir la emisión de GEI al capturar los gases generados y quemarlos; pero es necesario encontrar la opción más favorable entre las que se emplean para el manejo de desechos (vertederos, composteo, incineración y alimentación animal), de acuerdo con las propiedades de cada alimento. Por ejemplo, los desperdicios de OF son una opción en la alimentación de rumiantes (Pinos et al. 2010, del Razo et al. 2015), pero generan una cantidad considerable de $\mathrm{CH}_{4}$ de las 7 a las $12 \mathrm{~h}$ de incubación, si bien es menor $(\mathrm{p} \leq 0.05)$ que la generada por BOV y BOB. Así, el manejo adecuado de excedentes vegetales y sus desperdicios puede disminuir la emisión de $\mathrm{CH}_{4}$ a la atmósfera y promover la explotación de fuentes energéticas renovables (di María et al. 2015), no sólo a partir de los desperdicios vegetales que se depositan en los vertederos sino también donde éstos vegetales se cultivan. Según Eriksson et al. (2015) y Zebek et al. (2015), gran número de artículos y reportes coinciden en que la opción menos favorable para el manejo de desechos orgánicos y de residuos municipales es su deposición en vertederos, en tanto que la más favorable es la digestión anaerobia, ya que el material no digerido puede usarse como fertilizante por su contenido de nitrógeno y fósforo.

Los resultados del presente estudio coinciden con lo que argumentan Li et al. (2016), quienes afirman que los desperdicios de frutas y vegetales son de los sustratos más adecuados para la codigestión anaerobia y la generación de energía.

\section{CONCLUSIONES}

La generación de metano a partir de la fermentación anaerobia de desperdicios vegetales inoculados con líquido ruminal promueve la explotación de fuentes energéticas renovables. Sin embargo, se requieren más estudios para explorar condiciones que potencialicen su uso y pertinencia, y, en consecuencia, disminuir su deposición en vertederos y la emisión de gases efecto invernadero.

\section{AGRADECIMIENTOS}

Los autores agradecen a las autoridades de la Universidad Autónoma del Estado de Hidalgo las facilidades para realizar el presente estudio, a los estudiantes de Medicina Veterinaria y Zootecnia Rosa Elena Alfaro Díaz, Víctor Sánchez Oliver y Alba Lizbeth Reynoso Zacarías por su apoyo en las actividades de laboratorio, y a los Doctores Leluo Guan y Sergio S. González Muñoz por sus sugerencias.

\section{REFERENCIAS}

AOAC (1990). Official methods of analysis. 15a ed. Association of Official Analytic Chemists, Washington, D.C., 1094 pp.

Bonilla J.A. y Lemus C. (2012). Emisión de metano entérico por rumiantes y su contribución al calentamiento global y al cambio climático. Revisión. Rev. Mex. Cienc. Pecu. 3 (2), 215-246.

Cioabla A.E., Ionel I., Trif-Tordai G., Irimescu A. y Vetres I. (2013). Study on the quality of biogas obtained from agricultural residues during anaerobic fermentation. J. Environ. Prot. Ecol. 14 (1), 247-255.

del Razo O.E., Almaraz I., Espinosa V., Soriano R., Miranda L.A., Arias L., Guan L., Buendía G. y Peláez A. (2015). Comparative analysis of the in vitro fermentation of wasted cladodes (Opuntia spp.), lucerne and oat hays. S. Afr. J. Anim. Sci. 45 (5), 470-475.

DOI: 10.4314/sajas.v45i5.3

di Maria F., Sordi A., Cirulli G. y Micale C. (2015). Amount of energy recoverable from an existing sludge digester with the co-digestion with fruit and vegetable waste at reduced retention time. Appl. Energ. 150, 9-14.

DOI: 10.1016/j.apenergy.2015.01.146

Eriksson M., Strid I. y Hansson P.A. (2015). Carbon footprint of food waste management options in the waste hierarchy - a Swedish case study. J. Clean. Prod. 93, 115-125. DOI: 10.1016/j.jclepro.2015.01.026

FAO (2015). Pérdidas y desperdicios de alimentos en América Latina y el Caribe. Boletín 2, Oficina regional de las Naciones Unidas para la Alimentación y la Agricultura para América Latina y el Caribe. Food and Agriculture Organization, Santiago de Chile, $30 \mathrm{pp}$.

Islas-Espinoza M., de las Heras A., Vázquez-Chagoyán J.C. y Salem A.Z.M. (2017). Anaerobic cometabolism of fruit and vegetable wastes using mammalian fecal inoculums: fast assessment of biomethane production. J. Clean. Prod. 141, 1411-1418.

DOI: 10.1016/j.jclepro.2016.09.215 
Li K., Liu L.L.R. y Sun C. (2016). A review of methane production from agricultural residues in China. Renew. Sust. Energ. Rev. 54, 857-865. DOI: $10.1016 /$ j.rser.2015.10.103

Losada H., Martínez H., Vieyra J., Pealing R., Zavala R. y Cortés J. (1998). Urban agriculture in the metropolitan zone of Mexico City: Changes over time in urban, suburban and peri-urban areas. Environ. Urban. 10 (2), 37-54. DOI: 10.1177/095624789801000214

Losada H., Bennett R., Soriano R., Vieyra J. y Cortés J. (2000). Urban agriculture in Mexico City: functions provided by the use for dairy based livelihoods. Cities 17 (6), 419-431.

DOI: 10.1016/S0264-2751(00)00041-X

Pinos-Rodríguez J.M., Velázquez J.C., González S.S., Aguirre J.R., García J.C., Álvarez G. y Jasso Y. (2010). Effects of cladode age on biomass yield and nutritional value of intensively produced spineless cactus for ruminants. S. Afr. J. Anim. Sci. 40 (3), 245-250.

SAS. (2003). Statistical analysis software, v. 9.1.3. SAS Institute, Cary, NC., EUA.

Sánchez-Reyes C., Patiño-Iglesias M.E., Alcántara-Flores M.E., Reyes-Ortega Y., Pérez-Cruz M. y Ortíz-Muñoz E. (2016). Determinación del potencial bioquímico de metano (PBM) de residuos de frutas y verduras en hogares. Rev. Int. Contam. Ambie. 32 (2), 191-198. DOI: 10.20937/RICA.2016.32.02.05

Sauer M., Marx H. y Mattanovich D. (2012). From rumen to industry. Microb. Cell Fact. 11, 121.

DOI: $10.1186 / 1475-2859-11-121$

Scano E.A., Asquer C., Pistis A., Ortu L., Demontis V. y Cocco D. (2014). Biogas from anaerobic digestion of fruit and vegetable wastes: Experimental results on pilot-scale and preliminary performance evaluation of a full-scale power plant. Energ. Convers. Manage. 77, 22-30.

DOI: 10.1016/j.enconman.2013.09.004

Schofield P., Pitt R.E. y Pell A.N. (1994). Kinetics of digestion from in vitro gas production. J. Anim. Sci. 72, 2980-2991. DOI: 10.2527/1994.72112980x

SEDEMA (2015). Inventario de residuos sólidos. Ciudad de México 2015. Secretaría del Medio Ambiente del Gobierno de la Ciudad de México, Ciudad de México, $114 \mathrm{pp}$.

Sen B., Aravind J., Kanmani P. y Lay C.-H. (2016). State of the art and future concept of waste fermentation to bioenergy. Renew. Sust. Energ. Rev. 53, 547-557. DOI: 10.1016/j.rser.2015.08.065

Theodorou M.K., Williams B.A., Dhanoa M.S., McAllan A.B. y France J. (1994). A simple gas production method using a pressure transducer to determine the fermentation kinetics of ruminant feeds. Anim. Feed Sci. Technol. 48, 185-197. DOI: 10.1016/0377-8401(94)90171-6

Thi B.D., Biswarup S., Chin-Chao C., Gopalakrishnan K. y Chiu-Yue L. (2014). Food waste to bioenergy via anaerobic processes. Energy Proceed. 61, 307-312. DOI: 10.1016/j.egypro.2014.11.1113

Yan H., Zhao C., Zhang J., Zhang R., Xue C., Liu G. y Chen C. 2017. Study on biomethane production and biodegradability of different leafy vegetables in anaerobic digestion. AMB Express 7 (27).

DOI: $10.1186 / \mathrm{s} 13568-017-0325-1$

Zebek E., Szwejkowska M. y Raczkowski M. (2015). Legal and organisational solutions of municipal waste management in Poland in compliance with waste directive 2008/98/EC. J. Environ. Prot. Ecol. 16 (2), 652-658. 